\title{
Cranial Nerves VII To VIII
}

Currently a debate is raging as to whether, with the high-resolution scanning currently available, one needs to perform contrast-enhanced scans to exclude vestibular schwannomas in patients with sensorineural hearing loss (Allen et al., 1996; Chakeres and Schmalbrock, 1997; Curtin, 1997; El-Gammal, 1997; Hudgins, 1998; Jackler, 1996). This is a frequent indication for ordering studies in the elderly, and cost-containment considerations are often cited in the move to eliminate extra sequences and contrast agent expenses. Cranial nerves VII and VIII are well seen outlined by cerebrospinal fluid (CSF) in most patients and are the nerves most commonly involved with neurogenic neoplasms. The reasons why contrast-enhanced scans are still being performed are explained in this unit, but revolve around pathologies other than schwannomas of the nerves.

\section{IMAGING OF CRANIAL NERVES VII TO VIII}

Cranial nerves VII and VIII are usually affected by lesions in the cerebellopontine angle, internal auditory canal, and temporal bone. It is true, however, that cranial nerve VII then leaves the temporal bone to course through the parotid gland to innervate the muscles of facial expression. Therefore, though the typical evaluation of these nerves focuses on their intracranial and intraosseous portions, one must scrutinize the sagittal and coronal scans for the parotid glands, where lesions affecting the facial nerve may reside. In the case of congenital sensorineural hearing loss, a dedicated temporal bone study with high-resolution $T_{2}$-weighted scans may be contemplated.

Imaging of the cranial nerves requires a focused approach based on clinical symptomatology and signs. Thin-section imaging, high resolution, and contrast enhancement are essential components to this evaluation.

Table A7.3.1 lists the hardware necessary to perform the procedure, along with appropriate parameters. Standard head coil imaging is sufficient to obtain adequate scans on the intracranial portions of these cranial nerves. Anatomic imaging with high-quality fast spin echo $T_{2}$-weighted scan with the dark nerves outlined by bright CSF is required to see the portions of these nerves coursing through the cerebellopontine (CP) angle and internal auditory canal (IAC; Marsot-Dupuch et al., 1999; Iwayama et al., 1999). Gadolinium-enhanced scans are critical to the complete evaluation of these nerves, though the evaluation may be limited to sections through the lower pons and medulla, including the full range of the temporal bone. These scans should be centered on the internal auditory canal. No monitoring is required.

If a congenital source of hearing loss is the basis of the study, then one must know the scanner well enough to know whether surface coils will be required to obtain an adequate thin section evaluation of the cochlea, vestibule, semicircular canals, cochlear viaduct,

Table A7.3.1 Equipment Parameters for Cranial Nerve Imaging

\begin{tabular}{ll}
\hline Coil type & Head \\
Gradient coil strength & $25 \mathrm{mT} / \mathrm{m}$ \\
Flow compensation pulse & Yes, as needed \\
Peripheral gating & Not appropriate \\
Motion cushions & Useful \\
Use of contrast agents & Yes \\
\hline
\end{tabular}

BASIC PROTOCOL 
and vestibular aqueduct. Most 1.0 T or higher magnets will have adequate signal-to-noise ratio to allow the thin section scanning ( $2 \mathrm{~mm}$ or less) that is required for this indication. These scans will be performed at 512 by 512 matrices. At lower field strengths, or to optimize signal-to-noise ratio at higher field strengths, placing 5-in. round general-purpose surface coils over the ears may be advantageous. However for the traditional "rule out acoustic schwannoma," these extra preparations are not necessary.

\section{Materials}

Normal saline $(0.9 \% \mathrm{NaCl})$, sterile

Gadolinium-based contrast agent (e.g., Magnevist, Omniscan, or Prohance)

\section{Set up equipment and patient}

1. Interview (screen) the patient to ensure that he or she has no contraindications such as cardiac pacemakers or other implants containing ferromagnetic materials. Also be sure to find out if the patient has any health conditions that may require the presence of special emergency equipment during the scanning procedure, or necessitate any other precautions.

Generally standard screening forms are used for all patients scanned in a magnetic resonance system.

The presence of any ferromagnetic metals may be a health hazard to the patient when he or she is inside the magnet, and will also affect the imaging. If in doubt as to the exact composition of the items, it is best to exclude patients with any metal implants; see Shellock (1996) for discussion of what implants may be safely scanned using magnetic resonance.

Patients may be accompanied into the magnet room by a friend or family member, who can sit in the room during the scan and comfort the patient as needed. This companion must be screened as well to ensure the absence of loose metal objects on the body or clothing.

2. If the procedure is a research protocol, have the patient sign any necessary consent form.

3. Have the patient remove all jewelry and change into a gown to eliminate any metal that might be found in clothing.

4. Have the patient wash off any mascara and other makeup to avoid local tissue heating and image artifacts.

5. Inform the patient about what will occur during the procedure, what he or she will experience while in the magnet, and how to behave, including the following:

a. If earphones or headphones are used to protect the ears from the loud sounds produced by the gradients, the patient will be asked to wear these, but will be able to communicate with you at any time during the imaging.

b. The patient will be given a safety squeeze-bulb or similar equipment to request assistance at any time (demonstrate how this works).

c. For good results the patient should not talk, and should avoid or minimize swallowing or other movement, during each scan-i.e., as long as the banging sounds continue. Between scans, talking and swallowing are allowed in most cases, but should be avoided when comparative positional studies are being performed; the patient will be informed when this is the case.

d. Nevertheless, the patient may call out at any time if he or she feels it necessary.

6. Help the patient mount onto the table. Either before or right after the patient lies down, set up any triggering devices or other monitoring equipment that is to be used. 
7. Center the patient's head within the head coil and use the laser light to ensure symmetry. For most brain scans, placing the center landmark at the eyebrow level allows full coverage of the relevant regions of anatomy. Make sure that the head and neck are constrained to prevent motion, especially if high-resolution scans are to be run.

Generally the patient's head is fixed so that the head is horizontal (not tilted) and the neck and head lie along the axis of the patient table; other positions may be appropriate depending on the needs at hand.

8. If needed, place a pillow or other support under the knees to make the patient more comfortable.

9. Establish an intravenous line from which the contrast agent can be injected, and attach this line securely to the patient so that movement into or out of the magnet will not pull at the patient's arm.

It is preferable to insert the line prior to imaging and to leave the patient in the magnet, with no intervening motion, between the scans run before contrast agent injection and those run after injection.

10. Use the centering light to position the patient and put him or her into the center of the magnet.

Once this step has been performed, so long as the patient does not move on the table, the table itself can be moved and then replaced in the same position as before without jeopardizing the positioning of one scan relative to another.

11. If the patient is unable to hold still, provide an appropriate sedative.

\section{Sequence 1: Sagittal $T_{1}$-weighted spin echo scan}

Although a pilot scan can be acquired, it is not necessary here, because the first scan covers almost the entire brain in a sagittal fashion. These sagittal images can then be used as localizers for the application of saturation pulses when the transverse images are acquired.

12. Run sagittal $T_{1}$-weighted scan (see Table A7.3.2).

Saturation pulses are inferiorly placed. Often this scan best demonstrates the course of the 2nd genu and descending intramastoid portion of the facial nerve outlined by the air-filled mastoid bone. The exit from the stylomastoid foramen is well depicted, and the parotid glands are seen in their entirety when $5 \mathrm{~mm}$ interleaved scans are prescribed ear to ear. One can also assess the degree to which the temporal bones are fat-filled (marrow fat) and whether one needs to place fat-suppression pulses for post-gadolinium studies.

\section{Sequence 2: Transverse fast spin echo (FSE) scan}

13. Run transverse fast spin echo $T_{2}$-weighted scan (see Table A7.3.3).

Echo train length 12-32 depending on slices allowed and minimum echo spacing. Saturation pulses inferiorly and gradient moment nulling (flow compensation) should be applied. One now routinely applies frequency selective fat suppression pulses to produce a better dynamic range of contrast and to null the skull base and temporal bone fat. Acquisition time will be 3 minutes in length. Since hearing abnormalities may be affected along the full course of the auditory system, this study and the FLAIR that follows encompass the whole brain.

Sequence 3: Transverse FLAIR scan

14. Run transverse fluid attenuation inversion recovery (FLAIR) scan (see Table A7.3.4). 
Table A7.3.2 Sagittal $T_{1}$-Weighted Spin Echo Scan (Sequence 1) ${ }^{a}$

\begin{tabular}{ll}
\hline Patient position & Supine \\
Scan type & Spin echo \\
Imaging plane (orientation) & Sagittal \\
Central slice or volume center & Mid-brain \\
Echo time $\left(T_{\mathrm{E}}\right)$ & Minimum \\
Receiver bandwidth $(\mathrm{RBW})$ & $16 \mathrm{kHz}$ \\
Repeat time $\left(T_{\mathrm{R}}\right)$ & $400-600 \mathrm{msec}$ \\
Flip angle $(\mathrm{FA})$ & $90^{\circ}$ \\
Fields of view $\left(\mathrm{FOV}, \mathrm{FOV}_{\mathrm{y}}\right)$ & $240 \mathrm{~mm}, 240 \mathrm{~mm}$ \\
Resolution $(\Delta x, \Delta y)$ & $0.94 \mathrm{~mm}, 1.25 \mathrm{~mm}$ \\
Number of data points collected $\left(N_{x}, N_{y}\right)$ & 256,192 \\
Slice thickness $(\Delta z)$ & $5 \mathrm{~mm}$ \\
Number of slices & 28 \\
Slice gap & $0 \mathrm{~mm}$ \\
Number of excitations $(\mathrm{NEX})$ & 1 \\
Spatial saturation & Inferior \\
Slice series & Interleaved \\
Scan time & $\sim 2 \mathrm{~min}$ \\
\hline
\end{tabular}

${ }^{a}$ This sequence covers entire brain.

Table A7.3.3 Transverse Fast Spin Echo $T_{2}$-Weighted scan (Sequence 2) ${ }^{a, b}$

\begin{tabular}{ll}
\hline Patient position & Supine \\
Scan type & Fast spin echo \\
Imaging plane (orientation) & Transverse \\
Central slice or volume center & Mid-brain \\
Echo time $\left(T_{\mathrm{E}}\right)$ & $90-120 \mathrm{msec}$ \\
Receiver bandwidth (RBW) & $20 \mathrm{kHz}$ \\
Echo train length (ETL) & $12-32$ \\
Repeat time $\left(T_{\mathrm{R}}\right)$ & $3000-4000 \mathrm{msec}$ \\
Flip angle $(\mathrm{FA})$ & $90^{\circ}$ \\
Fields of view $\left(\mathrm{FOV}, \mathrm{FOV}_{\mathrm{y}}\right)$ & $240 \mathrm{~mm}, 240 \mathrm{~mm}$ \\
Resolution $(\Delta x, \Delta y)$ & $0.94 \mathrm{~mm}, 1.25 \mathrm{~mm}$ \\
Number of data points collected $\left(N_{x}, N_{y}\right)$ & 256,192 \\
Slice thickness $(\Delta z)$ & $5 \mathrm{~mm}$ \\
Number of slices & Variable \\
Slice gap & $0 \mathrm{~mm}$ \\
Number of excitations $(\mathrm{NEX})$ & 2 \\
Flow compensation & Yes \\
ZIP 512 & Yes, changes resolution to 0.47 \\
& mm by $0.63 \mathrm{~mm}$ \\
Chemical saturation & Yes, fat \\
Spatial saturation & Inferior \\
Slice series & Interleaved \\
Scan time & $\sim 3$ min \\
\hline
\end{tabular}

$a_{\text {This sequence covers entire brain. }}$

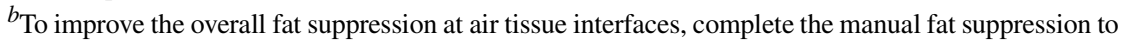
selectively suppress fat. 
Table A7.3.4 Transverse Fluid Attention Inversion Recovery (FLAIR) Scan (Sequence 3) $^{a}$

\begin{tabular}{ll}
\hline Patient position & Supine \\
Scan type & Inversion recovery fast spin echo \\
Imaging plane (orientation) & Transverse \\
Central slice or volume center & Mid-brain \\
Echo time $\left(T_{\mathrm{E}}\right)$ & $133 \mathrm{msec}$ \\
Receiver bandwidth (RBW) & $16 \mathrm{kHz}$ \\
Echo train length (ETL) & $12-32$ \\
Repeat time $\left(T_{\mathrm{R}}\right)$ & $8800 \mathrm{msec}$ \\
Inversion time $\left(T_{\mathrm{I}}\right)$ & $2200 \mathrm{msec}$ \\
Flip angle $(\mathrm{FA})$ & $180^{\circ}$ \\
Fields of view $\left(\mathrm{FOV}_{\mathrm{x}}, \mathrm{FOV}_{\mathrm{y}}\right)$ & $240 \mathrm{~mm}, 240 \mathrm{~mm}$ \\
Resolution $(\Delta x, \Delta y)$ & 0.94 \\
Number of data points collected $\left(N_{x}, N_{y}\right)$ & 256,256 \\
Slice thickness $(\Delta z)$ & $5 \mathrm{~mm}$ \\
Number of slices & Variable $(\sim 20)$ \\
Slice gap & $0 \mathrm{~mm}$ \\
Number of excitations $(\mathrm{NEX})$ & 1 \\
Flow compensation & Yes \\
ZIP 512 & Yes, changes resolution to 0.47 \\
& mm by $0.47 \mathrm{~mm}$ \\
Tailored RF & Yes \\
Spatial saturation & Inferior \\
Slice series & Interleaved \\
Scan time & $\sim 4-5$ min \\
\hline
\end{tabular}

${ }^{a}$ This sequence covers entire brain.

Echo train length 12-32 depending on slices allowed and minimum echo spacing. Saturation pulses inferiorly placed, tailored radiofrequency pulses and gradient moment nulling (flow compensation) are applied. The sections should be through the entire brain. Acquisition time will be 4 to 5 minutes in length.

Demyelinating disorders may lead to facial nerve and auditory symptoms, so one should use a sequence that does well at displaying the white matter lesions in the brainstem, infratentorial, and supratentorial compartments.

\section{Sequence 4: Transverse echo planar/diffusion weighted scan}

15. Run transverse echo planar/diffusion weighted imaging (see Table A7.3.5).

The minimum diffusion weighting should have a " $b$ " value of at least $1000 \mathrm{~s} / \mathrm{mm}^{2}$. These sections should be applied through the entire brain and aid in the detection of acute stroke processes.

\section{Sequence 5: Transverse $T_{2}$-weighted spin echo scan}

When there is a question of sensorineural hearing loss, one must evaluate the VII and VIII nerves with a very high-resolution imaging technique

16. Run a transverse heavily $T_{2}$-weighted fast spin echo (see Table A7.3.6).

One should employ fat saturation techniques, gradient moment nulling (flow compensation), and inferior placement of a spatial saturation pulse. 
Table A7.3.5 Transverse Echo Planar/Diffusion Weighted Imaging (Sequence $4)^{a}$

Patient position

Scan type

Imaging plane (orientation)

Central slice or volume center

Echo time $\left(T_{\mathrm{E}}\right)$

Receiver bandwidth (RBW)

Repeat time $\left(T_{\mathrm{R}}\right)$

Flip angle (FA)

Fields of view $\left(\mathrm{FOV}_{\mathrm{x}}, \mathrm{FOV}_{\mathrm{y}}\right)$

Resolution $(\Delta x, \Delta y)$

Number of data points collected $\left(N_{x}, N_{y}\right)$

Slice thickness $(\Delta z)$

Number of slices

Slice gap

Number of excitations (NEX)

Slice series

Scan time
Supine

Echo planar/diffusion weighted

image

Transverse

Mid-brain

Minimum

$62 \mathrm{kHz}$

$10,000 \mathrm{msec}$

$90^{\circ}$

$240 \mathrm{~mm}, 240 \mathrm{~mm}$

$1.88 \mathrm{~mm}, 1.88 \mathrm{~mm}$

128,128

$5 \mathrm{~mm}$

Variable ( 10)

$0 \mathrm{~mm}$

1

Interleaved

$1 \mathrm{~min}$

${ }^{a}$ This sequence covers entire brain.

Table A7.3.6 Question of Sensorineural Hearing Loss (Transverse Heavily $T_{2}$-Weighted Fast Spin Echo; Sequence 5) ${ }^{a}$

Patient position

Scan type

Imaging plane (orientation)

Central slice or volume center

Echo time $\left(T_{\mathrm{E}}\right)$

Receiver bandwidth (RBW)

Echo train length (ETL)

Repeat time $\left(T_{\mathrm{R}}\right)$

Flip angle (FA)

Fields of view $\left(\mathrm{FOV}_{\mathrm{x}}, \mathrm{FOV}_{\mathrm{y}}\right)$

Resolution $(\Delta x, \Delta y)$

Number of data points collected $\left(N_{x}, N_{y}\right)$

Slice thickness $(\Delta z)$

Number of slices

Slice gap

Number of excitations (NEX)

Flow compensation

Chemical saturation

Spatial saturation

Slice series

Scan time
Supine

Fast spin echo

Transverse

Internal auditory

canals

$200 \mathrm{msec}$

$24 \mathrm{kHz}$

24

4000-5000 msec

$90^{\circ}$

$180 \mathrm{~mm}, 180 \mathrm{~mm}$

$0.35 \mathrm{~mm}, 0.35 \mathrm{~mm}$

512,512

$2 \mathrm{~mm}$

Variable ( 20)

$0 \mathrm{~mm}$

4

Yes

Yes, fat

Inferior

Interleaved

$\sim 4 \mathrm{~min}$

\footnotetext{
${ }^{a}$ To improve the overall fat suppression at air tissue interfaces, complete the manual fat
} suppression to selectively suppress fat. 
Table A7.3.7 Transverse Post-Gadolinium $T_{1}$-Weighted Scan (Sequence 6)

Patient position

Scan type

Imaging plane (orientation)

Central slice or volume center

Echo time $\left(T_{\mathrm{E}}\right)$

Receiver bandwidth (RBW)

Repeat time $\left(T_{\mathrm{R}}\right)$

Flip angle (FA)

Fields of view $\left(\mathrm{FOV}_{\mathrm{x}}, \mathrm{FOV}_{\mathrm{y}}\right)$

Resolution $(\Delta x, \Delta y)$

Number of data points collected $\left(N_{x}, N_{y}\right)$

Slice thickness $(\Delta z)$

Number of slices

Slice gap

Number of excitations (NEX)

Flow compensation

Spatial saturation

Slice series

Scan time
Supine
Spin echo
Transverse
Internal auditory canals
Minimum
$16 \mathrm{kHz}$
400-600 msec
$90^{\circ}$
$180 \mathrm{~mm}, 180 \mathrm{~mm}$
$0.70 \mathrm{~mm}, 0.70 \mathrm{~mm}$
256, 256
$3 \mathrm{~mm}$
Variable $(\sim 20)$
$0 \mathrm{~mm}$
2-3
Yes
Inferior
Interleaved
$\sim 5-7$ min

Table A7.3.8 Coronal Post-Gadolinium Spin Echo $T_{1}$-Weighted Scan (Sequence 7) $^{a}$

\begin{tabular}{ll}
\hline Patient position & Supine \\
Scan type & Fast spin echo \\
Imaging plane (orientation) & Coronal \\
Central slice or volume center & Mid-brain \\
Echo time $\left(T_{\mathrm{E}}\right)$ & Minimum \\
Receiver bandwidth $(\mathrm{RBW})$ & $16 \mathrm{kHz}$ \\
Echo train length $(\mathrm{ETL})$ & $\leq 4$ \\
Repeat time $\left(T_{\mathrm{R}}\right)$ & $400-600 \mathrm{msec}$ \\
Flip angle $(\mathrm{FA})$ & $90^{\circ}$ \\
Fields of view $\left(\mathrm{FOV}, \mathrm{x}, \mathrm{FOV}_{\mathrm{y}}\right)$ & $180 \mathrm{~mm}, 180 \mathrm{~mm}$ \\
Resolution $(\Delta x, \Delta y)$ & $0.70 \mathrm{~mm}, 0.70 \mathrm{~mm}$ \\
Number of data points collected $\left(N_{x}, N_{y}\right)$ & 256,256 \\
Slice thickness $(\Delta z)$ & $3 \mathrm{~mm}$ \\
Number of slices & Variable \\
Slice gap & $0 \mathrm{~mm}$ \\
Number of excitations $(\mathrm{NEX})$ & $2-3$ \\
Flow compensation & Yes \\
ZIP 512 & Yes, changes resolution to 0.35 \\
& mm by $0.35 \mathrm{~mm}$ \\
Chemical saturation & Yes, fat \\
Spatial saturation & Inferior \\
Slice series & Interleaved \\
Scan time & $\sim 2-4$ min \\
\hline${ }_{\text {To improve the overall fat suppression at air tissue interfaces, complete the manual fat suppression to }}$ &
\end{tabular}


Sequence 6: Transverse post-contrast $T_{1^{-} \text {-weighted scan }}$

17. Leave the patient in the magnet, inject the contrast agent, and flush the line with 10 $\mathrm{ml}$ saline.

A dose of $0.1 \mathrm{mmol} / \mathrm{kg}$ of contrast agent is usually given.

18. Run transverse post-gadolinium $T_{1}$-weighted scan (see Table A7.3.7) through the internal auditory canals.

Saturation pulses inferiorly placed and gradient moment nulling (flow compensation) should be applied. These scans are performed without fat suppression (as opposed to the coronal scans that follow) so as to visualize the rare lipomas or hemorrhagic lesions that can affect the CP angle, IAC, and/or the temporal bone. One must be able to translate the transverse scans to the coronal scans so as to interpret the suppressed and nonsuppressed images accurately. If necessary, one can perform multiformatting to produce transverse pre-contrast studies from the sagittal 5-mm contiguous $T_{1}$-weighted first sequence and correlate the two. For some, it may be easier to perform transverse precontrast studies and eliminate this potential pitfall. If this is done, the parameters should not change from preto post-contrast studies, other than single average scans for precontrast sequence.

\section{Sequence 7: Coronal post-contrast $T_{1}$-weighted scan}

19. Run coronal post-gadolinium spin echo $T_{1}$-weighted scan (see Table A7.3.8).

Frequency-selective fat suppression should be applied. Spatial saturation pulses inferiorly placed and gradient moment nulling (flow compensation) are encouraged. The sections should include the whole of the temporal bone, brainstem, and the majority of the parotid gland.The sequence can be performed as a fast spin echo $T_{1}$-weighted scan, but the matrix should be ZIPped to 512 by $\times 512$, no more than an echo train length of 4 should be used, and fat suppression should be applied.

ALTERNATE PROTOCOL

Cranial Nerves VII To VIII

A7.3.8

\section{IMAGING OF CRANIAL NERVES VII TO VIII WHERE NON-NEOPLASTIC LESIONS ARE SUSPECTED}

\section{Demyelinating Disorders}

If demyelinating etiologies for the cranial nerve deficits are considered, then a more extensive $T_{2}$-weighted evaluation of the brain may be indicated. The post-gadolinium scans should be extended to cover the whole brain. If one identifies lesions that enhance and lesions that do not, it would imply a polyphasic disease such as multiple sclerosis.

\section{Congenital Sensorineural Hearing Loss}

As indicated previously, a more dedicated $T_{2}$-weighted study is required for those instances when congenital causes of sensorineural hearing loss are considered. In this case, high-resolution imaging should include slice thicknesses of less than $2 \mathrm{~mm}$ and matrices of 512 by 512. An advantage of using a head coil for these studies is that both ears can be imaged simultaneously with the brainstem. Often, surface coil imaging produces inadequate evaluation of the exit zones of the nerves, due to signal drop-off. While many institutions used a 3D-gradient echo sequence that allows sub-millimeter slice partitions (some as thin as $0.5 \mathrm{~mm}$ to $0.7 \mathrm{~mm}$ ), others are using multi-slice 2D fast spin echo scanning. We prefer 2D fast spin echo scans with $1.5-\mathrm{mm}$ thick images using a head coil to provide the best compromise of coverage, signal to noise, and scan timing (see Table A7.3.9). We use a $T_{\mathrm{R}}$ of 3000 to $5000 \mathrm{msec}, T_{\mathrm{E}}=80 \mathrm{msec}, \mathrm{ETL}=24,1.5 \mathrm{~mm}$ contiguous slices, 256 by 256 matrices ZIPped to 512 by 512 or 1024 by 1024, with 4-6 NEX. The in-plane matrices (number of data points collected) of the 3D GRASS (Gradient Recalled Acquisition in the Steady State) scans are usually limited to 256 by 256, though this is compensated by the use of smaller fields of view and surface coils images. The localization for these scans may require extra scout images. As for multi-slice $2 \mathrm{D}$ fast 
Table A7.3.9 2D Fast Spin Echo (Sequence 8) ) $^{a, b}$

\begin{tabular}{ll}
\hline Patient position & Supine \\
Scan type & Fast spin echo \\
Imaging plane (orientation) & Transverse \\
Central slice or volume center & Mid-brain \\
Echo time $\left(T_{\mathrm{E}}\right)$ & $180 \mathrm{msec}$ \\
Receiver bandwidth (RBW) & $32 \mathrm{kHz}$ \\
Echo train length (ETL) & 24 \\
Repeat time $\left(T_{\mathrm{R}}\right)$ & $3000-5000 \mathrm{msec}$ \\
Flip angle (FA) & $90^{\circ}$ \\
Fields of view (FOV, FOV & \\
Resolution $(\Delta x, \Delta y)$ & $240 \mathrm{~mm}, 240 \mathrm{~mm}$ \\
Number of data points collected $\left(N_{x}, N_{y}\right)$ & $0.94 \mathrm{~mm}, 0.94 \mathrm{~mm}$ \\
Slice thickness $(\Delta z)$ & 256,256 \\
Number of slices & $1.5 \mathrm{~mm}$ \\
Slice gap & Variable \\
Number of excitations (NEX) & $0 \mathrm{~mm}$ \\
Flow compensation & $4-6$ \\
ZIP 512 or ZIP 1024 & Yes \\
& Yes, changes resolution to 0.47 \\
ZIP 2 & mm, 0.47 mm \\
ZIP 4 & Not appropriate \\
Tailored RF & Not appropriate \\
Extended dynamic range $(E D R)$ & No \\
No phase wrap (NPW) & Yes \\
Chemical saturation & No \\
Spatial saturation & Yes \\
Scan time & Inferior \\
\hline$a_{\text {This sequence covers entire brain. }}$ & 8 min \\
${ }_{\text {To improve the overall fat suppression at air tissue interfaces, complete the manual fat suppression to }}$ \\
selectively suppress fat. & \\
\hline
\end{tabular}

spin echo scans, they do allow thinner slice partitions, but scan times can get prolonged with larger matrices, even with ZIPping.

\section{Sequence 8: $2 D$ fast spin echo}

1. Run sequence 8 according to Table A7.3.9.

\section{COMMENTARY}

\section{Background Information}

Because vestibular schwannomas are not the only cause of hearing loss or vertigo, one should not perform a high-resolution $T_{2}$-weighted scan (such as the one above) to screen for sensorineural hearing loss without also performing post-contrast $T_{1}$-weighted scans (Fulbright et al., 1995; Fitzgerald and Mark, 1998; Hudgins, 1998; Sartoretti-Schefer et al., 1999). Although it is probably true that over $90 \%$ of vestibular schwannomas may be identified with high quality $T_{2}$-weighted sequences, the post-contrast studies are critical to demonstrate labyrinthitis, neuritis, subarachnoid seeding, enhancement of the vestibular aqueduct (seen often in Menière's disease), labyrinthine schwannomas, temporal bone schwannomas, and some intracanalicular schwannomas (Ginsberg et al., 1996; Haught et al., 1998).

Hemifacial spasm is thought to be caused by vascular compression of the facial nerve. An MRA (MR angiography) (see Table A7.3.10) 
Table A7.3.10 MR Angiography to See Vascular Compression of Facial Nerve ${ }^{a, b}$

\begin{tabular}{|c|c|}
\hline Patient position & Supine \\
\hline Scan type & 3-D gradient echo \\
\hline Imaging plane (orientation) & Transverse \\
\hline Central slice or volume center & Internal auditory canals \\
\hline Echo time $\left(T_{\mathrm{E}}\right)$ & $6.9 \mathrm{msec}$ \\
\hline Receiver bandwidth (RBW) & $16 \mathrm{kHz}$ \\
\hline Repeat time $\left(T_{\mathrm{R}}\right)$ & $35 \mathrm{sec}$ \\
\hline Flip angle (FA) & $30^{\circ}$ \\
\hline Fields of view $\left(\mathrm{FOV}_{\mathrm{x}}, \mathrm{FOV}_{\mathrm{y}}\right)$ & $240 \mathrm{~mm}, 240 \mathrm{~mm}$ \\
\hline Resolution $(\Delta x, \Delta y)$ & $0.47 \mathrm{~mm}, 0.70 \mathrm{~mm}$ \\
\hline Number of data points collected $\left(N_{x}, N_{y}\right)$ & 512,256 \\
\hline Slice thickness $(\Delta z)$ & $1.0 \mathrm{~mm}$ \\
\hline Number of slices & Variable \\
\hline Slice gap & 0 \\
\hline Number of excitations (NEX) & 1 \\
\hline Flow compensation & Yes \\
\hline ZIP 512 & $\begin{array}{l}\text { Yes, changes resolution to } 0.23 \\
\mathrm{~mm} \text { by } 0.35 \mathrm{~mm}\end{array}$ \\
\hline ZIP 2 & Yes \\
\hline Extended dynamic range (EDR) & Yes \\
\hline Chemical saturation & Fat saturation \\
\hline Spatial saturation & Superior \\
\hline Magnetization transfer & Yes \\
\hline Slice series & Interleaved \\
\hline Scan time & $\sim 8 \min$ \\
\hline
\end{tabular}

should be added to the protocol centered at the internal auditory canal. One should scrutinize the raw data files to see the bright vessel impacting on the low signal intensity facial nerve.

There are many philosophies on how to screen or evaluate the patient with sensorineural hearing loss. In many instances the protocol will depend on the patient's age, the ancillary clinical findings, and the presence of other medical illnesses. Once again, an ounce of conversation with the patient or clinician may save a pound of scan time (or gadolinium).

If there are multiple cranial nerve deficits or bilateral hearing deficits, one might be dealing with neurofibromatosis type II, subarachnoid seeding on nerve roots, sarcoidosis, Lyme disease, granulomatous infections, etc., all of which require greater scrutiny of the post-contrast scans (Ginsberg et al., 1996; SartorettiSchefer et al., 1999; Smith et al., 1997). One might need to evaluate the whole brain for a source of drop metastases.

\section{Critical Parameters}

The patient must be reasonably symmetric in the head coil so that both internal auditory canals are in the same plane. This is critical for the high-resolution $T_{2}$-weighted scans, where little coverage is afforded by the longer scan times from the 512 by 512 matrix. The employment of multiple quick scouts will often position the anatomy perfectly. The authors have utilized a $T_{1}$-weighted FMPSPGR (fast multiplanar spoiled gradient echo: see Table A7.1.2) to quickly localize the IACs in patients who were difficult to position symmetrically. Adjust the imaging parameters to be $T_{\mathrm{R}}=100 \mathrm{msec}$, $T_{\mathrm{E}}=$ minimum, $\theta$ (flip angle) $=70^{\circ}, \mathrm{FOV}_{\mathrm{x}}=$ $\mathrm{FOV}_{\mathrm{y}}=20 \mathrm{~cm}$, slice thickness $4 \mathrm{~mm}$ with no gap, 512 phase encoding steps and 256 readout points. It may be necessary to run a few of these quick "scouts" in order to get the proper alignment. One can start with a transverse image and then oblique the following scouts from there. Marginal time employed with this will yield 
very good results, especially when comparing anatomic details coming in and out of plane on different slices. These techniques help produce a quicker and more confident interpretation.

\section{Troubleshooting}

Obtaining coronal scout images and obliquing the transverse scans to ensure both IACs are in plane together may be helpful steps. One can also use the transverse $T_{2}$-weighted brain images to determine the optimal center point for the transverse slices. If not having a pre-contrast $T_{1}$-weighted scan is problematic and the reformatted sagittal does not suffice, then one should just bite the bullet and call the patient back for an unenhanced scan after 12 hr.

Often, one of the most difficult tasks to master is complete fat saturation within the imaging volume. Typically, the overall fat suppression is at its worst when the imaging volume is adjacent to an air tissue interface causing local magnetic field inhomogeneities (i.e., secondary to metallic dental work). Although the scanners of today can often complete this task automatically, there is no better way of improving the fat suppressed image then by manually adjusting the center frequency and the position of the RF pulse that will impose the fat suppression.

While imaging post contrast, it is likely that flow artifacts over the imaging volume will significantly increase, making very subtle lesions difficult to assess. The authors have found that, with the simultaneous addition of gradient moment nulling and inferior spatial saturation pulses, the image quality improved drastically and the motion across the anatomy decreased However, with the addition of gradient moment nulling, an increase in the minimum $T_{\mathrm{E}}$ will typically result, and may change the $T_{1}$ weighting of the image. We have not found this slight increase in the effective $T_{\mathrm{E}}$ to be of any real clinical significance.

\section{Anticipated Results}

The post-contrast scans through the IACs will demonstrate nearly all vestibular schwannomas. As for the facial nerve, it is normal to see its geniculate ganglion horizontal tympanic portion vertical descending intramastoid portion enhance. The intracanalicular and labyrinthine portions prior to the first genu of the nerve should not enhance or else it is pathologic.

The high-resolution $T_{2}$-weighted scans should show the endolymph and perilymph in the cochlea, labyrinth, cochlear aqueduct, ves- tibular aqueduct and semicircular canals as bright structures amidst the dark temporal bone. The turns of the cochlea and the size of the aqueducts should be scrutinized.

\section{Time Considerations}

Since the post-contrast scans are the most valuable in this scenario, one can skimp on some signal averages, if necessary, to complete an uncooperative patient's study. Some fast recovery fast spin echo sequences create $T_{2}$ contrast with much shorter three digit $T_{\mathrm{R}}$ values, and could also be used to speed the patient along.

\section{Index of Terms}

The clinical imaging instructions and terminology utilized in this unit are primarily geared towards General Electric equipment. An Index of Terms is provided that should bridge the gap of vendor specific terminology.

Chemical saturation A technique that applies an additional radiofrequency (RF) pulse (at a desired distance from the center frequency) to selectively suppress a tissue. This technique can be utilized to suppress the signal from water, fat, or silicone.

Echo time $\left(\boldsymbol{T}_{\mathbf{E}}\right)$ The time that is measured from the initiation of the initial RF (radio frequency) pulse and the peak of the echo.

Echo train length (ETL) In fast spin echo or turbo spin echo imaging, the ETL will actually equal the number of echoes prescribed per $T_{\mathrm{R}}$. Successive $180^{\circ}$ refocusing pulses are applied to "rephase the dephasing" protons in an effort to maximize the number of lines of $k$ space per $T_{\mathrm{R}}$. The formula for scan time in relation to Fast Spin Echo imaging and ETL is as follows:

Scan time $=\left(T_{\mathrm{R}}\right) \times($ no. of phase

encoding steps/ETL) $\times(\mathrm{NEX})$

Extended dynamic range (EDR) An imaging-enhancement tool that will allow the utilization of 32-bit data processing as opposed to the standard 16-bit processor. EDR in this way should improve SNR and resolution, but will utilize twice as much memory as a conventional acquisition.

Flow compensation Sometimes more widely expressed as gradient moment nulling, this is a way in which the system places flowing or moving spins into "phase coherence" with stationary spins.

Inversion time $\left(\boldsymbol{T}_{\mathrm{I}}\right)$ With inversion recovery pulse sequences, typically the inversion time is the time from the first $180^{\circ} \mathrm{RF}$ pulse to
Head and Neck 
the center of the next $90^{\circ} \mathrm{RF}$ pulse. This inversion time will essentially "null" the desired tissue depending on how long or short the $T_{\mathrm{I}}$ (inversion time) selected is, and the $T_{1}$ relaxation time of the corresponding tissue.

No phase wrap (NPW) will prevent wrap around artifacts (also known as aliasing artifacts) in the phase encoding direction. NPW should only be used when necessary because of the following:

a. NPW doubles the FOV in the phase direction.

b. NPW essentially doubles the phase encoding steps (to maintain resolution).

c. With NPW, you must reduce the NEX by half in order to maintain scan time.

Number of excitations (NEX) is a factor that is utilized to calculate the overall scan time and will directly effect the SNR. NEX is essentially the number of times that data are sampled per acquisition. Note that increasing the NEX to achieve overall better SNR is a rather inefficient way to improve signal. Doubling the NEX from 2 to 4 will only yield a $40 \%$ increase in SNR while it doubles scan time. Take note of the formula for scan time:

Scan time $=\left(T_{\mathrm{R}}\right) \times($ no. of phase

encoding steps $) \times(\mathrm{NEX})$

Receiver bandwidth (RBW) The range of frequencies that the MRI scanner is actually "tuned" to receive. This will directly affect the overall SNR. This will not be done by increasing or decreasing the signal, but rather there will be an increase and/or decrease in the amount of noise received relative to the alteration of the RBW. An increase in the RBW will increase the range of frequencies that the scanner will evaluate and thus decrease the overall SNR. In comparison, utilizing a narrow bandwidth should yield less noise and improve the overall SNR. The relationship of the receiver bandwidth and SNR can be thought of as inversely proportional to the square root of the bandwidth.

Rectangular field of view (REC FOV) Asymmetric field of view (typically in the phase encoding direction). REC FOV is typically utilized when a body part is longer in one direction than another. By utilizing an asymmetric FOV, the system will not collect a portion of the data, thereby decreasing the scanning time.

Repetition time $\left(\boldsymbol{T}_{\mathbf{R}}\right)$ The time in a pulse sequence between successive excitation pulses.

Spatial saturation employs an additional RF pulse to cause moving spins within a deter- mined area to be selectively dephased. This application will reduce motion from flow and/or respiratory artifacts and will limit the number of slices per $T_{\mathrm{R}}$ in general. Since a spatial saturation pulse employs additional RF, which is to be deposited into the patient, special attention should be focused on the SAR (specific absorption rate). Today all MRI scanners have a program that internally monitors how much RF can be applied over a given period of time. This formula takes into account the patient's body weight. This actual body weight needs to be accurately input at all times for patient safety. An inappropriate weight will cause the improper limit of RF to be transmitted relative to a safe period of time in which this is to occur.

Tailored RF An imaging option that improves image quality on fast spin echo (FSE) sequences with relatively short $T_{\mathrm{E}}$ 's. Tailored $\mathrm{RF}$ will improve edge blurring by reducing overall echo spacing.

ZIP 512/ZIP 1024 Better known as "zerofill interpolation process," this is a reconstruction algorithm that allows the user to scan at a $256 \times 256$ matrix and then the data are zerofilled to a $512 \times 512$ matrix (or $1024 \times 1024$ respectively).

ZIP 2/ZIP 4 Slice ZIP essentially is also a "zerofill interpolation process" that will create additional slices through the interpolation procedure. These slices are created with an offset of $50 \%$ of the original imaging slice locations.

\section{Literature Cited}

Allen, R.W., Harnsberger, H.R., Shelton, C., et al. 1996. Low-cost high-resolution fast spin-echo MR of acoustic schwannoma: An alternative to enhanced conventional spin-echo MR? AJNR Am. J. Neuroradiol. 17:1205-1210.

Bigelow, D.C., Eisen, M.D., Smith, P.G., Yousem, D.M., Levine, R.S., Jackler, R.K., Kennedy, D.W., and Kotapka, M.J. 1998. Lipomas of the internal auditory canal and cerebellopontine angle. Laryngoscope 108:1459-1469.

Chakeres, D.W. and Schmalbrock, P. 1997. MR techniques for the internal auditory canal. AJNR Am. J. Neuroradiol. 18:1394-1395.

Curtin, H.D. 1997. Rule out eighth nerve tumor: Contrast-enhanced $T_{1}$-weighted or high-resolution $T_{2}$-weighted MR? AJNR Am. J. Neuroradiol. 18:1834-1838.

El-Gammal, T. 1997. MR techniques for the internal auditory canal. AJNR Am. J. Neuroradiol. 18:1393-1394.

Fitzgerald, D.C. and Mark, A.S. 1998. Sudden hearing loss: Frequency of abnormal findings on 
contrast-enhanced MR studies. AJNR Am. J. Neuroradiol. 19:1433-1436.

Fulbright, R.K, Erdum, E., Sze, G., and Byrne, T. 1995. Cranial nerve enhancement in the Guillain-Barré syndrome. AJNR Am. J. Neuroradiol. 16:923-925.

Ginsberg, L.E., De Monte, F., and Gillenwater, A.M. 1996. Greater superficial petrosal nerve: Anatomy and MR findings in perineural tumor spread. AJNR Am. J. Neuroradiol. 17:389-393.

Haught, K., Hogg, J.P., Killeffer, J.A., Voelker, J.L., and Schochet, S.S.J. 1998. Entirely intracanalicular meningioma: Contrast-enhanced MR findings in a rare entity. AJNR Am. J. Neuroradiol. 19:1831-1833.

Hudgins, P.A. 1998. Inner ear imaging: More than rule out acoustic. AJNR Am. J. Neuroradiol. 19:1807-1808.

Iwayama, E., Naganawa, S., Ito, T., Fukatsu, H., Ikeda, M., Ishigeki, T., and Ichinose, N. 1999. High-resolution MR cisternography of the cerebellopontine angle: $2 \mathrm{D}$ versus $3 \mathrm{D}$ fast spin-echo sequences. AJNR Am. J. Neuroradiol. 20:889895.

Jackler, R.K. 1996. Cost-effective screening for acoustic neuroma with unenhanced MR: a clini- cian's perspective. AJNR Am. J. Neuroradiol. 17:1226-1228.

Marsot-Dupuch, K., Dominguez-Brito, A., Ghasli, K., and Chouard, CH. 1999. CT and MR findings of Michel anomaly: Inner ear aplasia. AJNR Am. J. Neuroradiol. 20:281-284.

Sartoretti-Schefer, S., Kollias, S., and Valavanis, A. 1999. Ramsay Hunt syndrome associated with brain stem enhancement. AJNR Am. J. Neuroradiol. 20:278-80.

Shellock, F.G. 1996. Pocket Guide to MR Procedures and Metallic Objects. Lippincott-Raven, Philadelphia.

Smith, M.M., Thompson, J.E., Thomas, D., Castillo, M., Carrier, D., Mukherji, S.K., and Gilliam, D. 1997. Choristomas of the seventh and eighth cranial nerves. AJNR Am. J. Neuroradiol. 18:327-329.

Contributed by Robert W. Evers and David M. Yousem

The Johns Hopkins Hospital

Baltimore, Maryland 